\title{
A Mechanical Shocking Source Designed for Shallow Seismic Exploration
}

\author{
Xu Shuang-cheng \\ China University of Geosciences (Beijing) \\ Beijing, Country \\ Yu Xiang * \\ China University of Geosciences (Beijing) \\ Beijing, Country \\ Email: yuxiang@cugb.edu.cn \\ Zhang Qi-sheng \\ China University of Geosciences (Beijing) \\ Beijing, Country
}

\author{
Zhai Jin-hai \\ China University of Geosciences (Beijing) \\ Beijing, Country \\ Zang Hong-jiao \\ China University of Geosciences (Beijing) \\ Beijing, Country
}

\begin{abstract}
Shallow seismic exploration is the unique approach to obtain first-hand data of engineering geology for municipal construction. In this work, a mechanical shocking source has been designed for facilitating exploration of constructive stratum datum of urban construction. Aiming at the existing issues of three commonly used artificial seismic sources, this device is expected to meet three requirements: (i) Sufficient impact force to guarantee the credibility of the received signal. In view of weak impact energy of sledge hammer, we regulated the compression spring number and elastic coefficient for favorable excitation energy. (ii) Convenience and efficiency. In view of complex structure and expensive price of vibroseis, we adopted a simple and reliable system design to ensure a convenient operation. (iii) Safety and pollution-free. Impact hammer impacts stressed plate under the spring force and its own gravity, so as to avoid unsafe explosion and environmental pollution caused by the sharp chemical reactions.
\end{abstract}

Keywords-mechanical shocking source; urban construction; shallow seismic exploration; environmentfriendly; easy operation

\section{INTRODUCTION}

Shallow seismic exploration is the unique approach to obtain first-hand data of engineering geology for municipal construction, and artificial seismic source is the signal origin of the exploration ${ }^{[1,2]}$. The shallow refers to the exploration ranging from ground to 500-meter-deep strata, which is directly involved with urban construction projects ${ }^{[3]}$. Shallow seismic exploration utilizes trans-mission characteristic of seismic wave stimulated by artificial seismic source at strata interface to explain the engineering geological structure ${ }^{[4]}$. Municipal construction is profoundly affecting national economy and people livelihood. Using rail traffic for example, subway has become an optimal choice to solve the congestion of big cities, and high-speed trains make inter-city transportation more convenient ${ }^{[5-7]}$. Geological factors have important influence on safety of municipal construction. To systematically advance the urban construction, confirmation and implementation of building programs such as rail routes and building location depends on realtime and accurate geological date, so as to avoid underground latent faults, voids and abnormal objects ${ }^{[8]}$.

Exploring artificial seismic source suitable for urban construction is a cutting-edge subject. Artificial seismic source is core mechanism for shallow exploration, and its three commercial used sources are dynamite, vibroseis and sledge hammer. Seismic source of dynamite can readily produce an effective waveform due to its strong explosive impulse, but suffers from insecurity and environmental pollution ${ }^{[9,10]}$, especially in densely populated area. The frequency of vibroseis can be controlled, but it cannot be widely used due to its complex structure and high price. Sledge hammer has a low price and is easy to carry around, but suffers from weak impulse energy, narrow penetration depth, and intensive labor intensity ${ }^{[11]}$. As a result, this work attempts to design a shallow seismic exploration source suitable for municipal construction, and this design is expected to share three traits of large impulse energy, easy operation, and environment-friendly. 


\section{OVERALL FRAMEWORK OF SEISMIC EXPLORATION} DEVICE FOR SHALLOW ENGINEERING

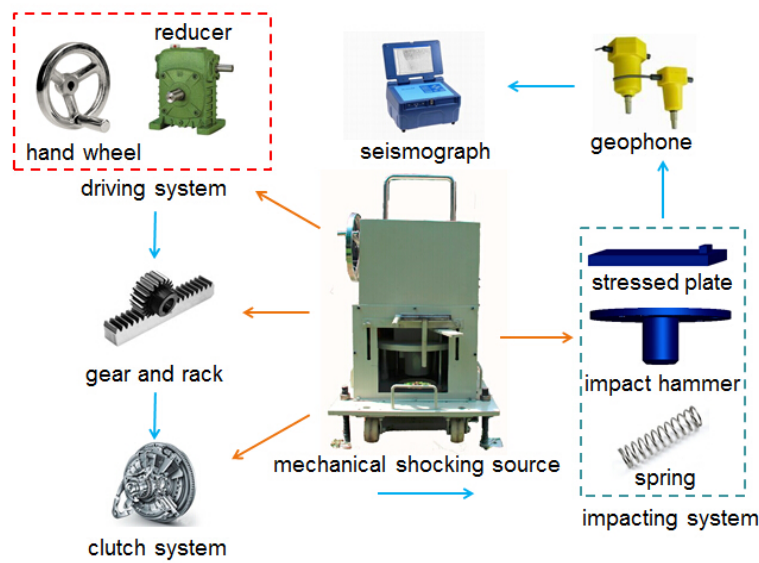

Figure 1. Overall framework of seismic exploration device for shallow engineering

Fig. 1 shows the overall framework of seismic exploration device for shallow engineering. Artificial earthquake is used for detecting characteristics of seismic strata in seismic exploration, and the artificial earthquake is often fulfilled through the artificial seismic source. The generated seismic waves propagate down through the ground, and the geophone on the ground transmits the received signal to the seismograph. In this way, mechanical shocking source is the core mechanism of the seismic exploration device, and is signal source for the exploration. Developing the shallow seismic exploration source needs to face the three issues: (i) Weak impulse energy of sledge hammer causes too weak signal; (ii) The structure of vibroseis is complex, and its cost is high; (iii) The dynamite is not safe, and its explosion pollutes the ambience. In this way, we attempts to design a mechanical shocking source, and it is expected to meet three requirements as follows: (i) Sufficient impact force to ensure the credibility of the receiver signal; (ii) Simple and reliable system design allows for a convenient and effective operation; (iii) Using impact system to fulfill device safety and pollution-free.

The blue arrow exhibits the order of operation sequence in Figure 1. Prior to operation, put stressed plate on the ground, and keep the mechanical shocking source at the ground-level state. During operation, operator clockwise rotates the hand wheel of driving system, and reducer drives gear rack to work. Rack moves up through gear driving, and clutch system moves up through rack pulling. As a result, energy-accumulating springs are compressed to accumulate energy from impacting system, and the hand wheel stops to rotate when clutch system comes to its required scale. During signal collection, impact hammer is separated from clutch system through adjusting angle of clutch spanner, the impact hammer impacts the stressed plate under action of its own gravity and accumulated elastic force of compression springs, and thus vibratory signal is generated through the impacting. Since different media have different wave impedances, the geophone receives returned signals from the strata and transmits it to the seismograph, constituting a work flow. Prior to next operation, operator counter-clockwise rotates the hand wheel to lock the clutch system, and may repeat the above work flow after receiving start command, so as to generate an admirable signal through the mechanical impact.

\section{SYSTEM STRUCTURE OF MECHANICAL SHOCKING} SOURCE

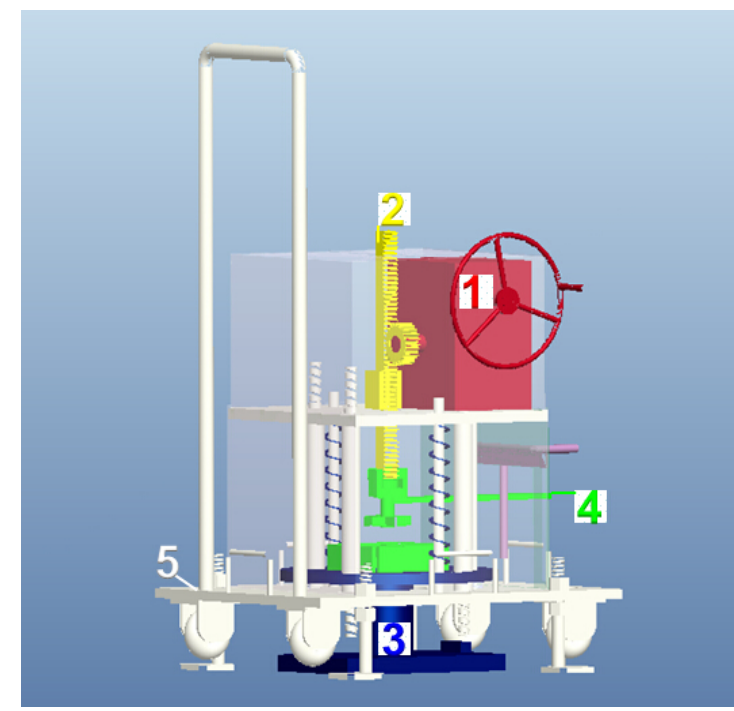

Figure 2. System structure of mechanical shocking source 1 Driving system; 2 Transmission system; 3 Impacting system; 4 Clutch system; 5 Auxiliary system

Fig. 2 shows systems structure of the mechanical shocking source. Working principle of the source is like this: for a flexible and convenient operation, operator can provide motive power through controlling driving system 1; transmission system 2 transfers the motive power to impacting system 3; impacting system 3 impacts stressed plate to generate an impacting signal; clutch system 4 controls closure and separation statuses of system 3; auxiliary system 5 matches other system connections and fixations.

\section{DESIGN DEMANDS AND SOLUTIONS OF MECHANICAL SHOCKING SOURCE}

\section{A. Sufficient impact force to ensure credibility of} receiver signal

Only good reliability of the received signal can give a clear picture for the stratum datum of urban construction. In this way, the mechanical shocking source needs to apply sufficient impact force to the ground for a reliable signal.

Energy analysis for the mechanical source is conducted according to the kinetic energy theorem:

$$
\frac{1}{2} m v^{2}=\frac{n}{2} k x^{2}+m g h
$$

Where, $m$ is quality of the impact hammer, $v$ is speed of the impact hammer, $k$ is coefficient of elasticity of springs, $x$ is deformation of the springs, $n$ is number of springs, $g$ is scaling factor of $9.8 \mathrm{~N} / \mathrm{kg}$, and $h$ is falling height of impact hammer.

Deducing from (1),

$$
v=\sqrt{\frac{n k x^{2}}{m}+2 g h}
$$

According to theorem of momentum: 


$$
F t=m v
$$

Where, $F$ means force of impact hammer on stressed plate, $t$ means time that the stressed hammer falls on the stressed plate.

It can be known from above calculations that, the mechanical shocking source can exert a high impact force of 2000-5000 $\mathrm{N}$ to the ground. The energy produced by such force can guarantee the credibility of the received signal.

\section{B. Simple and reliable system design allows for a convenient and effective operation}

In view of complex structure and high cost of vibroseis in urban construction, we attempt to introduce a simple and reliable driving system for solution. For a flexible operation and convenient movement, the rack transforms gear's rotary motion into reciprocating linear motion of transmission system; the wrench of clutch system controls impacting system statuses of closure and separation; and the auxiliary system works together with the other systems.

\section{1) Driving System}

Operator can provide motive power through controlling driving system, and the driving system is consisted of economic and applicable hand wheel and reducer. The hand wheel is fixed to the worm shaft of the reducer, and the reducer is mounted on the base plate. Operator can rotate the hand wheel readily. The reducer is a mechanism of gearing-down between driving link and working machine. The worm gear reducer we chose is a worm drive component enclosed in a rigid shell, and can provide large torque at low revolution speed. The reducer has a reverse self-locking function, and the teeth number of the gear of input shaft is less than that of output shaft, a certain transmission ratio can thus be guaranteed to reduce the labor intensity in evidence. In addition, the reducer can play a role of transmitting torque, since the input shaft and output shaft are neither on the same axis nor on the same plane.

In view of large physical exertion of existing driving system, our driving system consisting of the hand wheel and reducer can not only offer a convenient operation, but also avoid inverted phenomenon during rotation.

\section{2) Transmission System}

Transmission system is used to transmit the driving force of driving system. The gear, rack and rack seat of simple structure constitute the system. The gear and the worm shaft are connected by a flat key, and the gear engages with the rack. The upper end of rack is mounted on the rack seat, and the lower end is welded with clutch groove. The rack and gear have simple structures for steering. Different with synchronous belt and ball screw, they can either have any necessary travel length through splicing or transmit large power. In addition, the system can avoid skid phenomenon, and has a constant transmission ratio.

Using the gear and rack, this mechanism converts rotary motion into linear motion, and has three benefits of enough travel length, large transmission dynamic and constant transmission ratio.

3) Clutch system
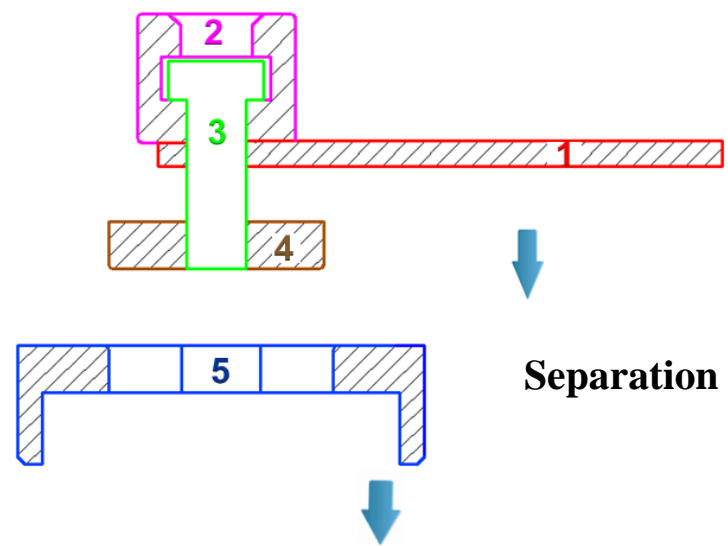

Separation

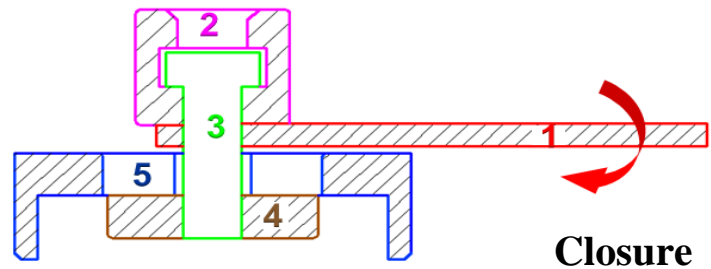

Figure 3. Schematic diagram of clutch system 1 Clutch spanner; 2 Clutch slot; 3 Clutch bearing; 4 Clutch lump; 5 Clutch plate

Fig. 3 shows two statuses of clutch system, which is used to control impacting system's closure and separation. The clutch system is mainly consisted of 1 clutch spanner, 2 clutch slot, 3 clutch bearing, 4 clutch lump, and 5 clutch plate. Clutch spanner 1 can be rotated flexibly in clutch slot 2; clutch bearing 3 is fixed in clutch slot 2; clutch lump 4 is welded on low-end of clutch bearing 3 . For a convenient and reliable operation, rotating clutch spanner 1 can adjust rotation angle of clutch lump 4 to select closure and separation statuses of clutch lump 4 and clutch plate 5 .

Prior to operation, the clutch system is in a status of separation; during operation, the clutch system is in a status of closure. Rotating hand wheel can make the device gather energy. When approaching specified value, rotating clutch spanner 1 lets the clutch system return to separation status for a shocking effect.

4) Auxiliary system

Auxiliary system is used to cooperate with other systems for easy operation and readily moving around. Auxiliary system is consisted of handrail, up-floor, downfloor, guiding shaft, supporting leg, truckle and outer cover. Guiding shafts are fixed between two floors through nuts; impact hammer passes through down-floor with a center bore and hammers the stressed plate.

Since the wild ground is not even, four supporting legs make the device in a steady status, where each leg is connected with down-floor through a pair of nuts to adjust the height. If the device needs to move, we can raise the supporting legs and truckles to get an easy and convenient movement.

\section{Using impact system to fulfill device safety and pollution-free}

The issues of dynamite source are: (i) Since the dynamite is not water-resistant, it may be easy to miss-fire because of water inflow from the broken shell or sealed part of dynamite; and it is easy to cause unsafe explosion 
because of large stress of the spiral charging as well as deformation of the medicine chamber and shell. (ii) The explosion process is a sharp chemical reaction, and its reaction products may pollute its surround environment.

As a solution, our impacting system is consisted of springs, impact hammer and stressed plate. During operating, springs are compressed under action of the driving and transmission system; the impact hammer welded with the clutch system will move up. Rotating clutch spanner lets impact system separate, and the impact hammer will impact the stressed plate under action of the springs force and the hammer gravity. The impact force can be adjusted by changing the elastic coefficient and the spring deformation.

\section{CONCLUSIONS}

Mechanical shocking source is the signal source of shallow seismic exploration. Three commonly used seismic sources for the exploration in the urban construction are: dynamite, vibroseis and sledge hammer. In view of the existing issues of the three commonly used sources, this paper proposes an overall framework of seismic exploration device for shallow engineering. This mechanical shocking source has five systems: driving system, transmission system, impacting system, clutch system, and auxiliary system.

This design has meet three expected requirements: (i) Sufficient impact force to ensure the credibility of the received signal. Springs can be compressed to accumulate energy under action of the driving system and transmission system; meanwhile, the compression spring number and elastic coefficient can be regulated for favorable excitation energy. (ii) Convenience and efficiency. Turning the hand wheel in driving system can lead reducer to run, and the rack makes gear's rotary motion change into reciprocating linear motion; the wrench of clutch system can control impacting system's closure and separation, and the device can readily move around with help of auxiliary system. (iii) Safety and pollution-free. Under action of own gravity of impact hammer and accumulated elastic force of compression springs, impacting the stressed plate can generate an admirable signal, so as to avoid unsafe explosion caused by the dynamite during forming shock waves and environ-mental pollution produced by the sharp chemical reactions.

\section{ACKNOWLEDGMENT}

This work was supported by the Fundamental Research Funds for Chinese Central Universities.

\section{REFERENCES}

[1] CHARLOTTE M K, ULRICH P, STEFAN T, et al. Sinkholes in the city of Hamburg-New urban shear-wave reflection seismic system enables high-resolution imaging of subrosion structures [J]. Journal of Applied Geophysics, 2012, 78: 133-143.

[2] KERIM M, JOSE A M. Urban seismic site investigations for a new metro in central Copenhagen: Near surface imaging using reflection, refraction and VSP methods [J]. Physics and Chemistry of the Earth, 2011, 36: 1228-1236.

[3] MARTI D, CARBONEL R, FLECHA I, et al. High-resolution seismic characterization in an urban area:Subway tunnel construction in Barcelona, Spain [J]. Geophysic, 2008, 73(2): B41B50.

[4] FENG Cheng, XIA Jiang-hai, XU Yi-xian, et al. A new passive seismic method based on seismic interferometry and multichannel analysis of surface waves [J]. Journal of Applied Geophysics, 2015, 117: 126-135.

[5] HUANG Chang-fu, XIA Yuan.Research on the role of urban rail transit in promoting economic development [J]. Procedia Engineering, 2011, 21: 520-525.

[6] YANG Jian-chun, DENG Zhi-hua. The interactive effect of Chinese logistics industry development and urbanization construction [C] // Proceedings of Seventh International Joint Conference on IEEE. Portugal: [s. n.], 2014: 143-146.

[7] VENKAT K, EIRINI K, DIMITRA P V, et al. An optimization model of energy and transportation systems: Assessing the highspeed rail impacts in the United States [J]. Transportation Research Part C, 2015, 54: 131-156.

[8] XU Kai, KONG Chun-fang, LI Jiang-feng, et al. Suitability evaluation of urban construction land based on geo-environmental factors of Hangzhou, China [J]. Computers and Geosciences, 2011, 37: 992-1002.

[9] MARGRAVE G F, BERTRAM M B, BERTRAM K L, et al. A low-frequency seismic field experiment [C] // Proceedings of SEG Annual Meeting. Society of Exploration Geophysicists, 2012 .

[10] EWALD L, DANIELA B, HELMUT G, et al. Transalp-deep crustal Vibroseis and explosive seismic profiling in the Eastern Alps [J]. Tectonophysics, 2006, 414: 9-38.

[11] WEI Zhou-hong. Design of a P-wave seismic vibrator with advanced performance [C] // Proceedings of 7th saudi society for Earth Science Conference. Portugal: [s. n.], 2008, 13(2): 123-136. 